The University of San Francisco

USF Scholarship: a digital repository @ Gleeson Library |

Geschke Center

$2-1-2000$

\title{
Simulation of dc Resistivity Data: Questioning Critical Scaling for the High-Tc Copper Oxides
}

Brandon R. Brown

University of San Francisco, brownb@usfca.edu

Follow this and additional works at: http://repository.usfca.edu/phys

Part of the Physics Commons

\section{Recommended Citation}

Brown, Brandon R., "Simulation of dc Resistivity Data: Questioning Critical Scaling for the High-Tc Copper Oxides" (2000). Physics and Astronomy. Paper 27.

http://repository.usfca.edu/phys/27 


\title{
Simulation of dc resistivity data: Questioning critical scaling for the high- $T_{c}$ copper oxides
}

\author{
Brandon Brown* \\ Department of Physics, University of San Francisco, San Francisco, California 94117
}

(Received 10 September 1999)

\begin{abstract}
We simulate de $V-I$ data using Gaussian distributions of depinning currents for the mixed state of a copper-oxide superconductor and subsequently submit the data to a critical scaling analysis. Though the simple model for simulation assumes no phase transition for the vortex matter, the generated data exhibit a collapse matching those in the experimental literature. The resulting would-be critical parameters depend on the simulated sensitivity level, reinforcing doubts concerning the utility of the scaling analysis for these systems.
\end{abstract}

The mixed state of high- $T_{c}$ materials has received intense scrutiny over the last decade. ${ }^{1}$ Considering an $H-T$ diagram, the mixed state below the irreversibility line has been described by (to name only three) a vortex glass for dense pointlike pinning, a Bragg glass for weak, dilute pinning, and a Bose glass for correlated pinning. ${ }^{2-4}$ The existence of the Bragg glass and the Bose glasses (both strong and weak) have gained strong support from magnetization measurements. ${ }^{5-7}$ However, support of the vortex glass has relied almost exclusively on $V-I$ data. ${ }^{8}$

For the copper oxides, dc $V-I$ data have typically been analyzed with critical scaling and interpreted in the context of a second-order vortex phase transition. Attempts to fit resistivity data with collective pinning and creep have proved difficult, especially since many different regimes of pinning (i.e., several orders of magnitude in applied current) can be covered by a single $V-I$ trace. ${ }^{9}$

Overall, the scaling collapses appear to lack universality in the copper oxides, undermining the conclusion that scaled data points to a second-order phase transition. Specifically, a systematic dependence of the critical parameters on the characteristic scale of vortex lattice dislocation density has been suggested. ${ }^{10}$ Such misgivings are compatible with theoretical studies that show no vortex glass-to-liquid transition can exist for finite temperatures, in two-dimensional (2D) or 3D cases, when the effects of screening are considered. ${ }^{11}$

At the same time, models of percolative depinning have suggested that dc $V-I$ characteristics can be described by distributions of depinning currents, and that the observed change in the shapes of $V-I$ isotherms is consistent with a percolative transition from a pinned vortex state for $T<T_{p}$ to a dissipative state for $T>T_{p}$ characterized by channels of vortex flow. ${ }^{12,13} \mathrm{We}$ note that percolative pictures of vortex depinning appear to be consistent with magnetization measurements which show plastic vortex response for a large region of the mixed state. ${ }^{14}$

Despite the advent of percolative models and the lack of demonstrated vortex glass universality, recent work continues to show scaling collapses for dc $V$ - $I$ data accompanied by the automatic claim of a second-order phase transition. ${ }^{15}$ Further investigation of the meaning of scaled data for these systems is warranted.

In this report, we simulate de resistivity data using a simple model of vortex depinning and subsequently submit the simulated data to a critical scaling analysis. We seek to answer two questions: does critical scaling apply to transport data in the case where a phase transition does not exist; and if a scaling analysis appears to collapse the data, are the derived critical exponents universal with respect to the experiment's sensitivity?

Some authors have modeled dc $V$ - $I$ data accurately assuming a highly parametrized form for critical-current distributions. ${ }^{12}$ Their work was based on distribution functions of pinned fluxoid clusters. Others have convincingly simulated $V-I$ traces from Gaussian distributions of depinning currents (corresponding to Gaussian distributions of pinning forces). ${ }^{16}$ This approach to vortex dynamics has enjoyed wider acceptance in low- $T_{c}$ literature than in high- $T_{c}$ literature. In particular, one effort treated a superconducting sample as a series of pinned vortex filaments, each with its own depinning current, such that the sample could be suitably described by a distribution function in current space. ${ }^{17}$ In this case, it is then reasonable to describe the voltage across a superconducting sample by

$$
V(I)=A \int_{0}^{I}\left(I-I^{\prime}\right) f\left(I^{\prime}\right) d I^{\prime},
$$

where $f(I)$ is a normalized distribution of depinning currents and the prefactor $A$ contains the specifics of the dissipative processes. Over all possible positive currents, $f(I)$ is normalized to unity.

We assume Gaussian depinning current distributions with centers $J_{0}$ and widths $\sigma$ varying linearly with temperature. This variation in temperature is zeroth order at best. It does not aim to most exactly match the phenomenological behavior of a vortex ensemble, and crucially it does not assume the sort of crossover in temperature expected for a phase transition.

In all, fifteen temperatures were simulated, spaced at 0.1 $\mathrm{K}$, between 90.0 and $91.4 \mathrm{~K}$. This roughly corresponds to a low-field (e.g., $0.25 \mathrm{~T}$ ) $V$ - $I$ window in $\mathrm{YBa}_{2} \mathrm{Cu}_{3} \mathrm{O}_{7-\delta}$ crystals, and the parameters of the first $(90.0 \mathrm{~K})$ distribution were chosen to roughly approximate experimental data of $\mathrm{YBa}_{2} \mathrm{Cu}_{3} \mathrm{O}_{7-\delta}{ }^{10}$ The distributions were normalized to represent a constant number of vortices for all temperatures. The form of the first distribution is given by

$$
f_{\text {sim }}=C \exp \left[\frac{-\left(J_{\text {sim }}-J_{0}\right)^{2}}{2 \sigma^{2}}\right] \text {, }
$$



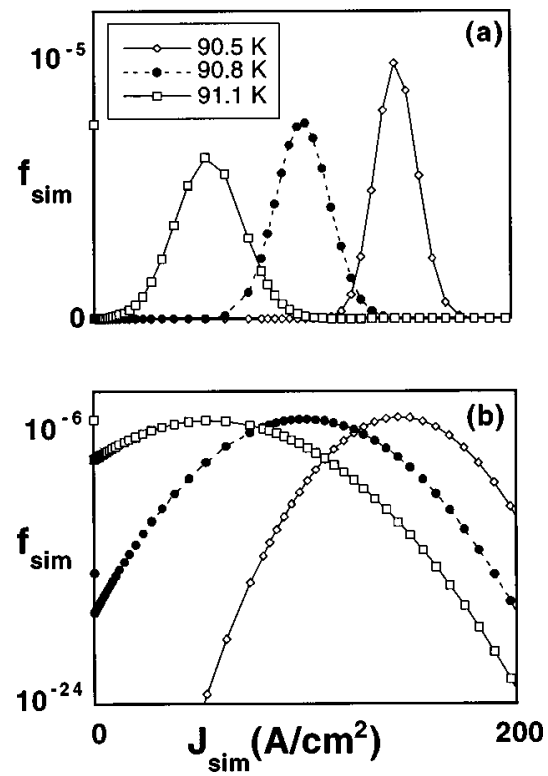

FIG. 1. Sample pinning distributions in (a) linear and (b) pseudolog representations.

where $s$ and $J_{0}$ were given temperature dependence. Specifically, they depend linearly on temperature via

$$
\begin{gathered}
\sigma(T)=5+10 \Delta T, \\
J_{0}(T)=220-150 \Delta T,
\end{gathered}
$$

in units of $\mathrm{A} / \mathrm{cm}^{2}$, where $\Delta T=T-90.0 \mathrm{~K}$. Selected distributions appear in Fig. 1. Simulated currents were spaced in a logarithmic fashion between $J_{\text {sim }}=0.01 \mathrm{~A} / \mathrm{cm}^{2}$ to $J_{\text {sim }}$ $=300 \mathrm{~A} / \mathrm{cm}^{2}$; this produces data that roughly match experimental data windows.

In the manner of other authors, we "pile" the number of vortices which would have been described by the $J<0$ area of the Gaussian in a distribution bin adjacent to $J=0 .{ }^{18} \mathrm{In}$ the simulation, this occurs for distributions corresponding to $T=90.7 \mathrm{~K}$ and above. Physically, if a pinning distribution is nonzero at $J=0$, a number of vortices will be depinned, as a delta function, at zero current. Samples of the distributions are depicted in Fig. 1; note the points at $J=0$ that correspond to a liquidlike vortex population.

Using Eq. (1) we simulated $E-J$ data from the distributions, assuming unity for geometrical factors, and $\rho$ - $J$ data where $\rho=E / J$. The resulting isotherms, shown in Fig. 2, provide a qualitative match of transport data for copperoxide superconductors. The isotherms between 90.0 and 90.5 are closely spaced and show an exponentially down-turning character. For 90.7-91.4 K the isotherms spread out and show finite linear resistivities. In addition, all isotherms show the flattening at high values of $J$, a signature of flux flow.

The critical scaling analysis for a vortex glass to vortex liquid phase transition has been described elsewhere. ${ }^{8}$ The first step involves finding a power-law isotherm at $T_{p}$ (or $T_{g}$ ) that divides exponentially downturning isotherms below (in theory corresponding to a superconducting glassy regime) from upturning isotherms above (corresponding to the resistive vortex liquid regime). The power-law slope at $T_{p}-n$ from $E \alpha J^{n}$ (also denoted $\alpha$ in the literature)-is directly

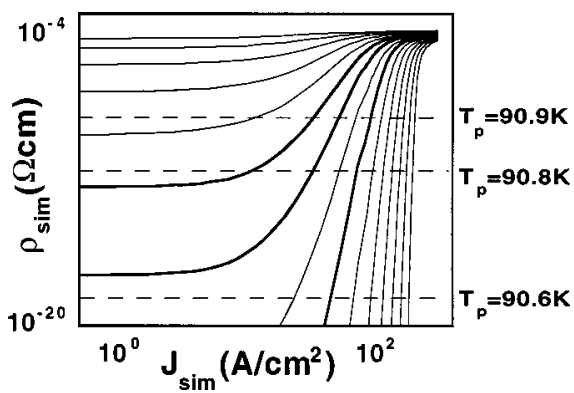

FIG. 2. Data simulated from Gaussian distributions of depinning currents for 90.0-91.4 K. Dashed lines denote the effect of different assumed sensitivities on the selection of $T_{p}$.

related to the dynamic critical exponent $z$, and this exponent should be universal given a set dimensionality. ${ }^{2}$

Before comparing the simulation to experimental data in more detail, we must choose a sensitivity, or a resistivity floor, at which to cut the data. For the case of a second-order phase transition, we would expect the same results independent of the sensitivity, but Fig. 2 clearly shows that the sensitivity choice is crucial for the simulated data. The three different sensitivities depicted in Fig. 2 each give a different value of $T_{p}$, using the same criteria that is used for analysis of the experimental data. It follows that the derived $n$ values vary as well, decreasing for decreasing sensitivity. Using the respective choices of $T_{p}$ for the three different sensitivity levels, we find $n=33$ for $T_{p}=90.6 \mathrm{~K}, n=16$ for $T_{p}=90.8 \mathrm{~K}$, and $n=10$ for $T_{p}=90.9 \mathrm{~K}$. In sum, lower sensitivity thresholds lead directly to lower values of $T_{p}$ and correspondingly higher values of $n$, regardless of where the underlying distributions first have a depinned population at $J=0$.

For presentation, we arbitrarily select a $\mathrm{pV} / \mathrm{cm}$ sensitivity for the simulated $E$ values. Proceeding with a scaling analysis, we derive $z=31$ from the power-law slope $(n=16)$ at $90.8 \mathrm{~K}$ and $\mathrm{V}=0.45$ by extracting an $s=\mathrm{V}(z-1)=13$ value from the temperature dependence of the linear resistivities. ${ }^{8}$ [These values do not match any in the literature, and we could certainly modify them by modifying the parameters in Eqs. (2) and (3). However, contriving the zeroth-order model is counter to our present purpose.] It is interesting to note that the linear resistivities extracted from this simulation give a power-law fit, a supposed signature of the vortex glass to liquid phase transition. As shown in Fig. 3, the data even

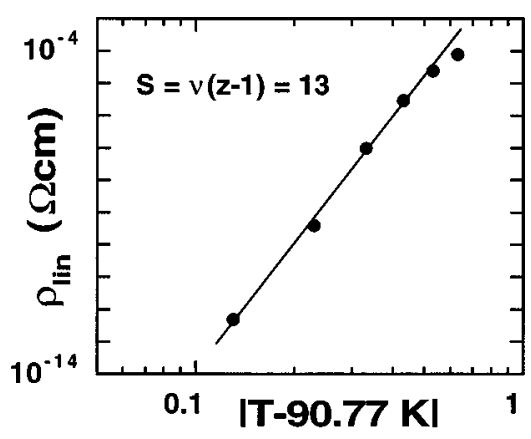

FIG. 3. Linear resistivities plotted versus $\left|T-T_{p}\right|$ for simulated data. According to the vortex glass theory, the slope gives $S$, and data that deviate from the power-law at high $T$ fall outside the critical scaling regime (Ref. 2). 


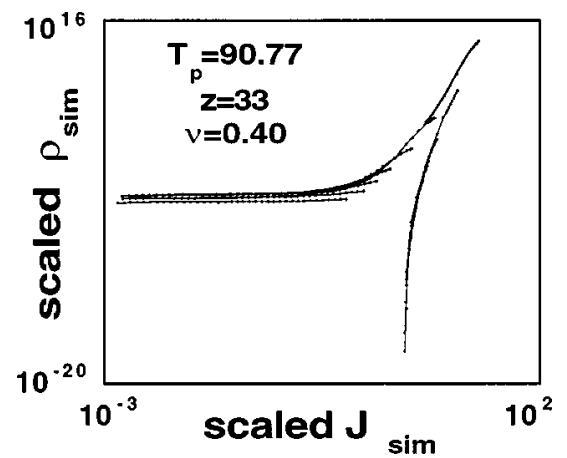

FIG. 4. Scaled data from the simulation with derived critical parameters.

bend away from the power-law at higher temperatures, just as one observes in the data of copper oxides. ${ }^{8}$

After conservatively cutting some of the flux flow data, the isotherms are scaled according to a vortex glass analysis, assuming a 3D system. In brief, the data are collapsed onto two universal functions $F \pm$ (corresponding to data above and below the suggested phase transition) by plotting $\rho_{\text {scale }}$ $=\rho_{0}{ }^{-1} \rho\left|1-T / T_{p}\right|^{\mathrm{v}(z-1)}$ and $J_{\text {scale }}=J_{0}{ }^{-1} J T^{-1}\left|1-T / T_{p}\right|^{-2 \mathrm{v}}$ where $\rho_{0}$ and $J_{0}$ have been set to one. ${ }^{8}$ Figure 4 shows the collapsed data and the results of optimizing the collapse by changing the parameters in a manner consistent with $s$ $=\mathrm{v}(z-1)$ as per the vortex glass theory. The deviations from the two branches occur for the remnant flux flow data and for the $91.4 \mathrm{~K}$ data. The deviation of $91.4 \mathrm{~K}$ matches data that would be considered outside the scaling regime in a vortex glass analysis, corresponding to the deviation of the linear resistivity point for $91.4 \mathrm{~K}$ in Fig. $3{ }^{8}$ Similar collapses, with different parameters derived from $n$, follow for the other two sensitivity levels.
The results answer our two original questions. Though no assumption of a vortex phase transition has been used in generating this simplified resistivity data, critical scaling collapses the data with a quality matching collapses that appear in the literature. The model generating this data is in fact so simple that it includes no explicit vortex interactions whatsoever. In addition, the resulting parameters depend demonstrably on the sensitivity level of the simulated experiment. This last point echoes the original misgivings of Esquinazi concerning the critical scaling of dc $V-I$ data. ${ }^{19}$ We add that the derived critical parameters would obviously vary with the Gaussian parameters in Eq. (3) were we to vary them. Broader distributions lead to lower values of $n$, and so forth, matching experimental results that examine current distributions. $^{10,18}$

We do not mean to question the existence of second-order vortex phase transitions in the mixed state; recent evidence for both the Bose glass and Bragg glass has been convincing. ${ }^{6,7}$ We comment only on the utility of dc resistivity data for studying vortex dynamics. Our results lead us to believe that critical scaling collapses in the copper oxides could just as easily represent convenient yet meaningless parameterizations as they could represent signatures of true phase transitions. The flexibility of the three parameter fit $\left(T_{p}, z\right.$, and $\left.\mathrm{v}\right)$ should not be underestimated. If the model of depinning-current distributions is appropriate for modeling the data-and we note that even the zeroth-order model used here qualitatively describes experimental data-then a scaling collapse may simply divide two different regimes of curvature in the underlying distributions.

This work was in large part supported by the NSF under DMR 9408628. We are grateful to J. Tate, A. L. Wasserman, and W. H. Warnes for helpful discussions.
*Electronic address: brownb@usfca.edu

${ }^{1}$ Exhaustive reviews of the literature are provided by G. Blatter et al., Rev. Mod. Phys. 66, 1125 (1994); E. H. Brandt, Rep. Prog. Phys. 58, 1465 (1995).

${ }^{2}$ D. S. Fisher, M. P. A. Fisher, and D. A. Huse, Phys. Rev. B 43, 130 (1991).

${ }^{3}$ T. Giamarchi and P. Le Dousal, Phys. Rev. B 52, 1242 (1995); T. Giamarchi and P. LeDousal, Phys. Rev. Lett. 76, 3408 (1996).

${ }^{4}$ D. R. Nelson and V. M. Vinokur, Phys. Rev. Lett. 68, 2398 (1992); Phys. Rev. B 48, 13060 (1993).

${ }^{5}$ D. Krusin-Elbaum et al., Phys. Rev. Lett. 72, 1914 (1994).

${ }^{6}$ S. Kokkaliaris et al., Phys. Rev. Lett. 82, 5116 (1999).

${ }^{7}$ A. W. Smith et al., Phys. Rev. B 59, R11 665 (1999).

${ }^{8}$ R. H. Koch et al., Phys. Rev. Lett. 63, 1511 (1989); J. M. Roberts et al., Phys. Rev. B 49, 6890 (1994); W. Lang et al., Z. Phys. B: Condens. Matter 100, 13 (1996); M. Friesen et al., Phys. Rev. B
54, 3525 (1996) (this list is representative of the data for YBCO films only).

${ }^{9}$ M. Leghissa et al., Z. Phys. B: Condens. Matter 92, 163 (1993);

R. Hiergiest and R. Hergst, Phys. Rev. B 55, 3258 (1997).

${ }^{10}$ B. Brown et al., Phys. Rev. B 55, R8713 (1997).

${ }^{11}$ C. Wengel and A. P. Young, Phys. Rev. B 54, 6869 (1996).

${ }^{12}$ K. Yamafuji and T. Kiss, Physica C 258, 197 (1996).

${ }^{13}$ M. Ziese, Physica C 269, 35 (1996).

${ }^{14}$ Y. Abulafia et al., Phys. Rev. Lett. 77, 1596 (1996).

${ }^{15}$ A. Sawa et al., Phys. Rev. B 58, 2868 (1998).

${ }^{16}$ R. Wördenweber, Phys. Rev. B 46, 3076 (1992).

${ }^{17}$ W. H. Warnes and D. C. Larbalestier, Appl. Phys. Lett. 48, 1403 (1986).

${ }^{18}$ H. S. Edelman and D. C. Larbalestier, J. Appl. Phys. 74, 3312 (1993).

${ }^{19}$ P. Esquinazi, Solid State Commun. 74, 75 (1990). 